Adhien, P., Dijk, L. van, Vegter, M. de, Westein, M., Nijpels, G., Hugtenburg, J.G. Evaluation of an pilot study to influence medication adherence of patients with diabetes mellitus type-2 by the pharmacy. International Journal of Clinical Pharmacy: 2013, 35(6), 1113-1119

\begin{tabular}{|l|l|}
\hline $\begin{array}{l}\text { Postprint } \\
\text { Version }\end{array}$ & 1.0 \\
\hline Journal website & http://link.springer.com/article/10.1007\%2Fs11096-013-9834-4 \\
\hline Pubmed link & $\underline{\text { http://www.ncbi.nlm.nih.gov/pubmed/23942987 }}$ \\
\hline DOI & $10.1007 / \mathrm{s} 11096-013-9834-4$ \\
\hline
\end{tabular}

This is a NIVEL certified Post Print, more info at http://www.nivel.eu

\title{
Evaluation of a pilot study to influence medication adherence of patients with diabetes mellitus type-2 by the pharmacy
}

\author{
PREM AdHIEN ${ }^{1,2,}$ LISET VAN DIJK ${ }^{3,}$ MARINKE DE VEGTER ${ }^{4}$ MARNIX WESTEIN ${ }^{5,}$ GIEL \\ NIJPELS ${ }^{1,2}$ AND JACQUELINE G. HUGTENBURG ${ }^{2,6}$
}

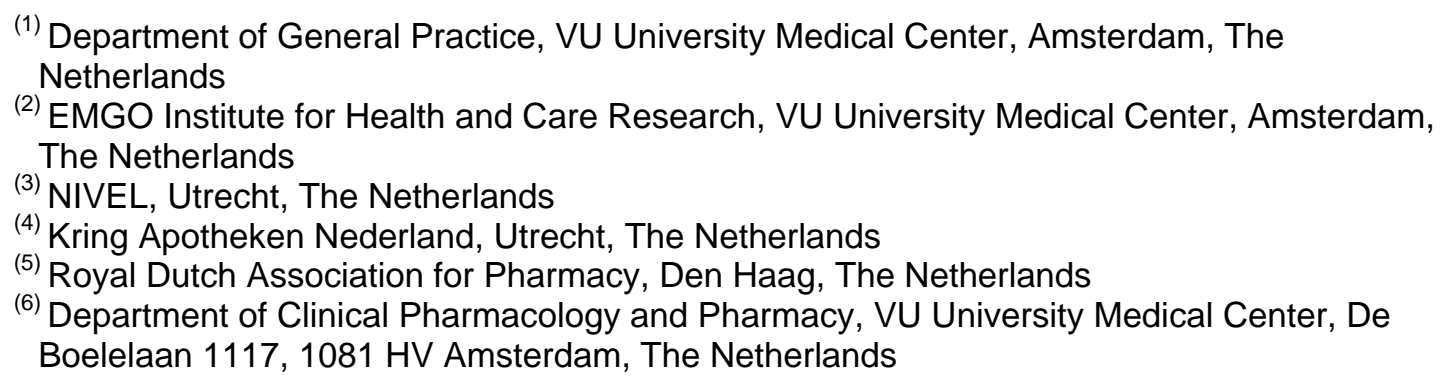

\begin{abstract}
Background Interventions aimed to increase adherence to drug treatment usually are not tailored to the needs of individual patients. A modular pharmacy intervention, named 'Support for Diabetes', was developed to improve adherence to type 2 diabetes treatment. Objective To evaluate the implementation of a new care intervention by using focus groups including pharmacy teams, and assess patient satisfaction. Setting Community pharmacies in The Netherlands Method The intervention comprises a structured patient interview, an intervention guide and modular interventions tailored to the underlying cause of non-adherence. Feasibility was studied in non-adherent type 2 diabetes mellitus patients, and evaluated by means of focus group interviews with pharmacists and pharmacy technicians. Topics included practicability of the patient selection procedure, patient interviews, materials developed for the intervention and general practitioner (GP) co-operation. Patients' experiences (n = 36) were assessed by means of a questionnaire. Main outcome measure Feasibility of the intervention and patients' satisfaction. Results Pharmacists and pharmacy technicians considered the intervention feasible and appreciated its pro-active approach. Involvement of pharmacy technicians proved a stimulating factor. Poor co-operation with GPs and lack of time as well as financial compensation were interfering factors. Patients appreciated the intervention and
\end{abstract}


Adhien, P., Dijk, L. van, Vegter, M. de, Westein, M., Nijpels, G., Hugtenburg, J.G. Evaluation of ar pilot study to influence medication adherence of patients with diabetes mellitus type-2 by the pharmacy. International Journal of Clinical Pharmacy: 2013, 35(6), 1113-1119

reported to follow the advice of pharmacists. Conclusion The 'Support for Diabetes' intervention is feasible to implement in pharmacy practice. Poor cooperation between pharmacists and GPs and lack of re-imbursement are obstructions for implementation on a wider scale. These issues should receive attention of pharmacists, policymakers and researchers.

\section{IMPACT OF FINDINGS ON PRACTICE STATEMENTS}

- It is feasible to implement a complex intervention for pharmaceutical care in daily pharmacy practice.

- Poor co-operation between pharmacists and GPs and lack of reimbursement are major barriers impeding the implementation of more complex pharmaceutical care interventions in daily practice.

\section{INTRODUCTION}

Type 2 diabetes mellitus (T2DM) is associated with an increased risk of complications like cardiovascular disease, retinopathy, nephropathy and peripheral vascular disease. Apart from lifestyle recommendations, intensive treatment with oral glucose lowering agents, insulin and medication dealing with (cardio-)vascular disease risk factors, should control blood glucose levels and decrease the risk of disease-related complications $\left[{ }^{1}\right]$. However, in $15-30 \%$ of T2DM patients adherence to drug treatment is suboptimal $\left[{ }^{2}, 3\right]$. Non-adherence is associated with higher $\mathrm{HbA1c}$ levels, higher diastolic and systolic blood pressure and higher cholesterol levels $\left[{ }^{4}\right]$ and has been shown to result in increased hospitalisation, mortality and health care costs $\left[{ }^{4}-6\right]$.

Patients are both intentionally and unintentionally non-adherent. Intentional nonadherence can be seen as a process in which the patient actively decides not to follow treatment recommendations. This attitude reflects a rational decision-making process in which the pros and cons of using or not using the prescribed medicines are weighed $\left[^{7}\right]$. Patients' beliefs and level of cognition are important determinants of this process $\left[{ }^{7}-{ }^{10}\right]$. Unintentional non-adherence refers to non-planned behaviour (e.g. forgetting to take pills) and is less strongly associated with beliefs and the level of cognition than intentional non-adherence $\left[{ }^{8},{ }^{10}\right]$.

Experience and knowledge of side effects, perceived ineffectiveness of treatment and lack of motivation are important causes of intentional non-adherence $\left[{ }^{3},{ }^{11}\right]$. Nonintentional adherence may result from forgetfulness and insufficient knowledge of the medication and its use $\left[{ }^{8}\right]$. Interventions aimed at improving non-adherence are often ineffective largely because they usually do not take into account the specific needs of individual patients and the various causes underlying non-adherence $\left[{ }^{5},{ }^{11}\right.$

$\left.{ }^{13}\right]$. Therefore, a tailored and modular intervention protocol focused on the causes of non-adherence and including the patient perspective was developed to support community pharmacists in their counselling of T2DM patients. The intention was to solve any problems encountered in close co-operation with the general practitioners (GPs) involved. Since the intervention concerns a working method that is new to community pharmacies, eventual factors interfering with the implementation will be identified. 
Adhien, P., Dijk, L. van, Vegter, M. de, Westein, M., Nijpels, G., Hugtenburg, J.G. Evaluation of ar pilot study to influence medication adherence of patients with diabetes mellitus type-2 by the pharmacy. International Journal of Clinical Pharmacy: 2013, 35(6), 1113-1119

\section{AIM OF THE STUDY}

To evaluate the implementation of a modular intervention to improve adherence of T2DM patients to drug treatment by using focus groups including pharmacy teams. Factors stimulating and impeding the implementation process were studied.

Secondary aim was to assess patient satisfaction with the activities of the pharmacist performed in the context of the intervention.

\section{METHODS}

\section{Participating pharmacies}

Four pharmacies in the Rotterdam area participated in the study. Each pharmacy enrolled 10 patients. Two pharmacies were located in the inner city of Rotterdam. Two other pharmacies were located in smaller towns in the Rotterdam area.

\section{Intervention 'Support for Diabetes'}

The intervention Support for Diabetes consists of the following four elements:

1. Preparatory workshops.

2. Selection of non-adherent patients by means of a web-based search developed by Dutch Foundation for Pharmaceutical Statistics (SFK).

3. A structured patient interview by a pharmacy technician in order to:

a. Identify the cause(s) of non-adherence.

b. Select the intervention module best suited to the patient's individual situation on the basis of the intervention protocol.

4. Follow-up by means of periodic telephone consultations.

\section{Ad 1: Preparatory workshops}

Before conducting the intervention, pharmacy personnel involved in the project participated in two interactive, 1 day workshops featuring two elements: (1) a training exploring the backgrounds of non-adherence by the research team (PA and $\mathrm{JH}$ ), and (2) a training in communicative skills in order to improve motivational interviewing techniques by an experienced communication trainer specialized in motivational interviewing in health care settings. The intervention and its working procedures were also explained.

\section{Ad 2: Selection of patients eligible for the intervention}

All four pharmacies used the standard search method developed by SFK for selecting non-adherent patients (as available to SFK data supplying pharmacies and pharmacies subscribing to SFK services). The SFK search strategy for non-adherent T2DM patients defines non-adherence as any discontinuation or interruption of antiT2DM medication which includes oral glucose lowering agents, antihypertensive drugs and statins. For patients who used a particular drug for less than 3 months, 3-6 months and more than 6 months, an interruption was defined as no use for a period longer than 8,27 and 55 days, respectively. Use was considered discontinued when there was no filling record for a period of 30 days or more. A switch to a drug of the same therapeutic class (e.g. enalapril to losartan) was not considered as nonadherence. 
Adhien, P., Dijk, L. van, Vegter, M. de, Westein, M., Nijpels, G., Hugtenburg, J.G. Evaluation of an pilot study to influence medication adherence of patients with diabetes mellitus type-2 by the pharmacy. International Journal of Clinical Pharmacy: 2013, 35(6), 1113-1119

Each pharmacy search produced a list of non-adherent patients eligible for participation. Patients younger than 18 years of age and those unable to communicate with the pharmacist were excluded. Patients wrongly classified as being nonadherent, for example because medicines were filled by another pharmacy or because the patient stayed for a certain period in a nursing home or hospital, were also not included. Each pharmacy randomly selected ten T2DM patients from the list to participate in the study. They were invited by telephone.

\section{Ad 3a: Individual structured interview to identify reasons for non-adherence}

To obtain insight into the causes of non-adherence a structured patient interview was conducted in the pharmacy by a pharmacy technician. The interview was based on the validated Medication Adherence Rating Scale (MARS) developed by Horne and co-workers $\left[{ }^{14}\right]$ and specifically addressed five major reasons for non-adherence; (1) lack of motivation to take the prescribed medication; (2) doubt about the effectiveness of the current treatment; (3) side effects; (4) problems in swallowing or injecting medication; (5) forgetting to take medication. In the case that (specific) non-adherence was detected the issue was further elaborated in the interview.

\section{Ad 3b: Intervention protocol in order to select an intervention module best suited to} the patient's individual situation

The 'Support for Diabetes' intervention guide and intervention protocol are used to select an intervention tailored to the cause(s) of non-adherence. These documents list the most common causes of non-adherence (see above) and contain five specific interventions (modules) addressing the major causes of non-adherence. They are directed: (1) to improve the 'motivation' of the patients to take the medicines as prescribed, i.e. counselling on the basis of the principles of Motivational Interviewing; (2) to address the problem that 'the current treatment does not work': e.g. in consultation with the GP, another dosage or drug is chosen and/or counselling of the patient on the effects and effectiveness of the medicines prescribed; (3) to address the problem of 'side effects': counselling of the patient on perceived and possible side effects and/or in consultation with the GP, lowering of the dosage or selection of an alternative drug; (4) to clarify and/or facilitate the taking of the medication, e.g. difficulties with swallowing tablets can be solved by packing tablet fragments in a capsule or grinding a tablet into a powder; and, (5) to offer tools to help patients 'remember' to timely use their medicines, e.g. proposing the use of a 'Baxter' roll, a week box or an alarm system.

\section{Ad 3: Follow-up}

Three and 6 months after the interview and intervention the medication record of each patient is evaluated by the pharmacist. Patients still not taking their medicines on time are offered telephone support or continued counselling. Protocols of this intervention have been published on the website of the Royal Dutch Foundation for Pharmacy (KNMP): http://www.knmp.nl/samenwerken-kennis-delen/ projectheek/projecten/2010/knmp-beroepsontwikkeling/Therapietrouw\%20Diabetes.

\section{Testing the feasibility of 'Support for Diabetes' intervention}

Since the Support for Diabetes intervention is a newly-developed tool intended for common use in Dutch community pharmacies, a feasibility study was performed. 
Adhien, P., Dijk, L. van, Vegter, M. de, Westein, M., Nijpels, G., Hugtenburg, J.G. Evaluation of ar pilot study to influence medication adherence of patients with diabetes mellitus type-2 by the pharmacy. International Journal of Clinical Pharmacy: 2013, 35(6), 1113-1119

The main body of this study consisted of focus group interviews with all participating pharmacists and pharmacy technicians involved (joint sessions). An initial focus group discussion was held during the intervention period and a second one a month after the intervention period. In health services research focus group discussions have been successfully used in studies exploring professional responses to changing management arrangements or improve professional development $\left[{ }^{15}\right]$. In our study we were interested in the response of the pharmacy teams to the implementation process of the 'Support for Diabetes' intervention. A topic list was developed to guide the focus of the group discussions. Since patient selection by means of the SFK-search and the structured patient interviews were major elements of the intervention, the focus group discussions concentrated on the practicability of the selection procedure and intervention techniques applied. In addition, GP co-operation and the preconditions for the wider implementation of this intervention were evaluated as were the materials provided to the pharmacies. Pharmacy personnel of all four pharmacies participated in the same focus group. Topics were introduced by the discussion leader, both researcher and practising community pharmacist (PA) who also led the discussions. The meetings (about $2 \mathrm{~h}$ each) were recorded. Recordings were transcribed verbatim in order to have the full text of the discussions available. Texts were categorized by two researchers (PA and $\mathrm{MdV}$ ) using a framework approach. To this end a framework of coded issues was developed. As such, the analytical process was explicit and informed by a priori reasoning $\left[{ }^{16}\right]$. MdV and PA independently coded the focus group interviews. Inconsistencies were discussed until agreement on the coding was reached. The feasibility study also comprised a short written patient questionnaire. Patients were asked to value the intervention with regard to its various components: the invitation to participate, the interview, and the measures proposed to address their non-adherence. They were also asked whether they considered the counselling of the pharmacist beneficial to their treatment.

\section{RESULTS}

\section{Participating pharmacies and patients}

Four pharmacies ( 4 pharmacists and 4 pharmacy technicians) were asked to participate and all agreed. However, one pharmacy withdrew after the start of the study and was replaced by another pharmacy. The pharmacies were situated both in a large city and in smaller towns nearby. The city pharmacies mainly cared for immigrants, whereas the peripheral pharmacies predominantly cared for patients of Dutch origin. Non-adherent patients were selected by applying the SFK-selection tool. Thirty-six patients were randomly chosen and invited to participate in the study: $32(88.9 \%)$ were willing to participate. Reasons for non-response were: the intervention was not deemed necessary and patients were too sick or on vacation. Of these 32 patients, $11(34.3 \%)$ were non-Western immigrants and $20(62.5 \%)$ were men. The intervention proved to be difficult to conduct. The completion of the project had a delay of 6 months instead of the expected 2 months.

\section{Focus group interview with pharmacists}

\section{Selection of patients and inviting the patients to participate}


Adhien, P., Dijk, L. van, Vegter, M. de, Westein, M., Nijpels, G., Hugtenburg, J.G. Evaluation of ar pilot study to influence medication adherence of patients with diabetes mellitus type-2 by the pharmacy. International Journal of Clinical Pharmacy: 2013, 35(6), 1113-1119

The pharmacists were positive about the method to select patients. It was easy, took little time and generally non-adherent patients were found. Pharmacists were advised always to check the data in the pharmacy information system before inviting a patient to participate. Two pharmacists had actually done this and indicated that the check took more time than using the SFK selection tool. It appeared that one patient was wrongly selected (quote 1 in Table 1).

\section{[TABLE 1]}

\section{Inviting the patients to share a conversation}

The pharmacist or the pharmacy technician invited patients by telephone, which took about five min. per patient. One of the pharmacy technicians indicated that it was sometimes difficult to reach the patients because their phone number was not known. Furthermore, one of the pharmacists had doubts about directly calling a patient (quote 2 in Table 1). Another pharmacist suggested approaching patients by means of a written invitation (quote 3 in Table 1). However, the overall experience was that patients appreciated the invitation by the pharmacist. Three pharmacists considered the leaflet to explain the intervention clear. The fourth pharmacist found the leaflet too confrontational and had it modified according to his own insights.

\section{Conversations with patients}

Pharmacy technicians were positive about the patient interviews. The interviews lasted 20-30 min. Pharmacy technicians observed that patients, in general, were very prepared to talk about their medication and that they appreciated the measures proposed to tackle their non-adherence and other problems with their medication (quote 4 in Table 1). They highly valued the structured interview protocol (quote 5 in Table 1). The modular intervention protocol was considered to be clear and useful for addressing various drug related problems.

\section{Communication with the GPs}

The intervention protocol recommends informing the GP about the implementation of a specific intervention. Only two of the pharmacists followed this recommendation. They reported to have a good relationship with the GP in their practice area. The other pharmacists did not inform GPs (quote 6 in Table 1). They only would consult a GP in the case of a specific drug problem, such as a drug-drug interaction or a contra-indication for the use of a newly prescribed drug. However, in case these pharmacists would consult GPs about a drug problem detected by means of a medication review or the intervention studied, these pharmacists suppose that the GPs would not co-operate. In contrast, one pharmacist suggested that directly calling the GP to discuss medication problems and measures to address these problems would be very useful in conducting the intervention.

\section{Stimulating factors}

The pharmacy teams were asked which factors encouraged them to continue with the intervention. The fact that the pharmacy technician played an important role in the intervention appeared of great value to the pharmacists (quote 7 in Table 1). 
Adhien, P., Dijk, L. van, Vegter, M. de, Westein, M., Nijpels, G., Hugtenburg, J.G. Evaluation of ar pilot study to influence medication adherence of patients with diabetes mellitus type-2 by the pharmacy. International Journal of Clinical Pharmacy: 2013, 35(6), 1113-1119

Furthermore, the pharmacy teams appreciated the positive response of their patients. They felt that the patients meant that the intervention had an added value above the usual care (quote 8 in Table 1).

\section{Interfering factors}

The pharmacy teams experienced problems in the implementation of the intervention. They specifically complained about a lack of time and shortage of staff. In addition, they reported that under these conditions the lack of financial compensation impeded the implementation of the intervention more widely.

\section{Patient experiences}

Patients were asked about their experiences with the service offered by means of a questionnaire, which was filled out by $25(78.1 \%)$ out of 32 patients. Although the number of patients was small, we performed a descriptive analysis on the data obtained (Table 2). Most patients appreciated the fact that the pharmacy invited them to share a conversation. One of them wrote that he had had many conversations with the pharmacist and thanked his pharmacist for this. In general, the patients found T2DM treatment support important and were of the opinion that they had benefited from the intervention. Most patients indicated that they would follow the recommendations to improve adherence.

\section{[TABLE 2]}

\section{DISCUSSION}

The present study shows that a modular and structured intervention to support nonadherent patients with medication problems is feasible to perform by community pharmacists in daily pharmacy practice. Already in 2003 the WHO stressed the necessity of tailoring interventions to the patients' needs and developed models for individualised interventions $\left[^{5}\right]$. Based on these models Herborg and co-workers described an intervention programme that included different strategies for distinguishing various types of non-adherence and a toolbox of instruments to tailor a counselling programme for patients individually $\left[{ }^{17}\right]$. More recently, Dimatteo and co-workers presented their Information-Motivation-Strategy concept, which distinguishes three major categories of non-adherence: patients do not understand what they have to do, patients are not motivated, and patients have no strategy to follow treatment recommendations $\left[{ }^{18}\right]$. Interventions should therefore be tailored to the specific causes of non-adherence $\left[{ }^{18}\right]$. It seems reasonable to assume that tailored and individualised counselling is more effective than conducting simple interventions. However, as yet the effectiveness of this approach has not been tested in a randomised clinical trial.

According to the pharmacists, patients appreciated that the pharmacists took time for them, answered their questions and supported them in addressing their medication problems. This was confirmed by the patients themselves. A previous study also showed that, according to patients, pharmacists should play a more prominent role in their medication management $\left[{ }^{19}\right]$.

Some of the pharmacists reported to have no good relationship with the GPs of their patients. This was considered an obstacle in conducting the intervention. Since good 
Adhien, P., Dijk, L. van, Vegter, M. de, Westein, M., Nijpels, G., Hugtenburg, J.G. Evaluation of ar pilot study to influence medication adherence of patients with diabetes mellitus type-2 by the pharmacy. International Journal of Clinical Pharmacy: 2013, 35(6), 1113-1119

co-operation between pharmacists and GPs is of great importance for the optimal drug treatment of patients $\left[{ }^{20}{ }^{23}\right]$, strategies to improve collaboration between health carers should be explored. According to the pharmacists, staff shortages and a lack of time also interfered with the timely implementation of the intervention in the study. In spite of a positive opinion of the pharmacists and pharmacy technicians on the 'Support for Diabetes' intervention and benefits expected for patients, it took about 8 months to complete the study, instead of 2 months. In particular, pharmacists appeared to have difficulties to organise the daily work routine in the pharmacy in such a way that there was sufficient time to provide more advanced pharmaceutical care on a continuous basis. It is also possible that other activities had a higher priority. The pharmacists themselves reported that both a lack of time and financial compensation were factors limiting the feasibility of the intervention. Adequate reimbursement by health insurers for pharmacy interventions aimed at improving the quality of pharmacotherapy of patients may change this situation. A limitation of the study is that the pharmacists and pharmacy assistants may have given socially desirable answers. Furthermore, the interventions carried out by the pharmacists were not evaluated. To get insight into the effects of the intervention on adherence and clinical outcome, a randomised controlled study involving a large number of patients should therefore be carried out.

\section{CONCLUSION}

The newly-developed 'Support for Diabetes' modular intervention proved feasible to implement in pharmacy practice and was appreciated by patients not-adherent to T2DM drug treatment. However, poor co-operation between pharmacists and GPs, and lack of re-imbursement are obstructions for a wider implementation of the intervention among community pharmacies. These problems should be addressed by pharmacists, policymakers and researchers.

\section{Acknowledgments}

The four pharmacy teams who participated in the study are acknowledged for their co-operation. The authors received unrestricted grants from Apotex, Regifarm, and the Royal Dutch Association for Pharmacy (KNMP).

\section{Funding}

None.

\section{Conflicts of interest}

None.

\section{REFERENCES}

1.Baan CA, Poos MJJC. Hoe vaak komt diabetes mellitus voor en hoeveel mensen sterven eraan. RIVM 2005.

2.Herings RMC, et al. Chronische Farmacotherapie Voorgezet-Een onderzoek naar de omvang, determinanten en mogelijke economische consequenties van het vroegtijdig afbreken van chronische farmacotherapie, PHARMO Instituut 2002. 
Adhien, P., Dijk, L. van, Vegter, M. de, Westein, M., Nijpels, G., Hugtenburg, J.G. Evaluation of ar pilot study to influence medication adherence of patients with diabetes mellitus type-2 by the pharmacy. International Journal of Clinical Pharmacy: 2013, 35(6), 1113-1119

3. Haynes RB, Ackloo E, Sahota N, McDonald HP, Yao X. Interventions for enhancing medication adherence (Review). Cochrane Database Syst Rev. 2008; (2):CD000011.

4.Ho PM, Rumsfeld JS, Masoudi FA, McClure DL, Plomondon ME, Steiner JF, Magid DJ. Effect of medication nonadherence on hospitalization and mortality among patients with diabetes mellitus. Arch Intern Med. 2006;17:1836-41.

5.Sabaté E. Adherence to long-term therapies. Evidence for action. Geneva: World Health Organization; 2003.

6.Lee WC, Balu S, Cobden D, et al. Prevalence and economic consequences of medication adherence in diabetes: a systematic literature review. Manag Care Interface. 2006;19:3141.

7.Wroe AL. Intentional and unintentional nonadherence: a study of decision-making. J Behav Med. 2002;25:355-72.

8.Lehane E, McCarthy G. Intentional and unintentional non-adherence: a comprehensive framework for clinical research and practice? A discussion paper. Int J Nurs Stud. 2007;44:1468-77.

9.Lowry KP, Dudley TK, Oddone EZ, Bosworth HB. Intentional and unintentional nonadherence to antihypertensive medication. Ann Pharmacother. 2005;39:1198-203.

10.Brown KK, Rehmus WE, Kimball AB. Determining the relative importance of patient motivations for nonadherence to topical corticosteroid therapy in psoriasis. J Am Acad Dermatol. 2006;55:607-13.

11. Hugtenburg JG, Blom ATG, Kisoensingh SU. Initial phase of chronic medication use; patients' reasons for discontinuation. Br J Clin Pharmacol. 2006;61:352-4.

12.Vermeire E, Wens J, Van Royen $\mathrm{P}$, et al. Interventions for improving adherence to treatment recommendations in people with type 2 diabetes mellitus. Cochrane Database Syst Rev. 2005; (2):1-37.

13.Sluijs EM, Van Dulmen S, Van Dijk L, et al. Patient adherence to medical treatment: a meta review. Utrecht: NIVEL; 2006.

14. Horne R, Weinman J. Patients' beliefs about prescribed medicines and their role in adherence to treatment in chronic physical illness. J Psychosom Res. 1999;47:555-67.

15.Wong LP. Focus group discussion: a tool for health and medical research. Singapore Med J. 2008;49:256-60.

16.Pope C, Ziebland S, Mays N. Qualitative research in health care. Analysing qualitative data. BMJ. 2000;320:114-6.

17. Herborg H, Haugbølle LS, Sørensen L, et al. Developing a generic, individualised adherence programme for chronic medication users. Pharm Pract. 2008;6:148-57.

18.DiMatteo MR, Haskard-Zolnierek KB, Martin LM. Improving patient adherence: a threefactor model to guide practice. Health Psychol Rev. 2012;6:74-91.

19. Tinelli M, Ryan M. Bond C Patients' preferences for an increased pharmacist role in the management of drug therapy. Int J Pharm Pract. 2009;17:275-82.

20.Beney J, Bero LA, Bond C. Expanding the roles of outpatient pharmacists: effects on health services utilisation, costs, and patient outcomes (Cochrane Review). Cochrane Lib. 2001; (2):2003.

21.Krska J, Cromarty JA, Arris F, et al. Pharmacist-led medication review in patients over 65: a randomized, controlled trial in primary care. Age Ageing. 2001;30:205-11.

22.Bernsten C, Bjorkman I, Caramona M, et al. Improving the well-being of elderly patients via community pharmacy-based provision of pharmaceutical care: a multicentre study in seven European countries. Drugs Aging. 2001;18:63-77.

23.Sellors J, Kaczorowski J, Sellors C, et al. A randomized controlled trial of a pharmacist consultation program for family physicians and their elderly patients. Can Med Assoc J. 2003;169:17-22. 
Adhien, P., Dijk, L. van, Vegter, M. de, Westein, M., Nijpels, G., Hugtenburg, J.G. Evaluation of a pilot study to influence medication adherence of patients with diabetes mellitus type-2 by the pharmacy. International Journal of Clinical Pharmacy: 2013, 35(6), 1113-1119

\section{TABLES}

Table 1: Quotes from pharmacists, pharmacy technicians and patients

Quotes related to selecting and inviting patients to participate

1. A quote from one pharmacist: "I invited a patient who switched from pravastatin to ezetrol, another drug to reduce cholesterol. According to the SFK search this patient was non-adherent, but in reality she was known as loyal to therapy"

2. One pharmacist had doubts about calling patients: "you tell someone that he or she is not taking the medication properly. You are trying to bring that message across tactfully. I wonder what a patient thinks about this invitation."

3. One pharmacist proposed a different approach: "Choose a different approach and tell the patient that we want to help make better use of the medicines, that we pursue optimal product use so that patients live longer."

Quotes related to the conversations with the patients

4. One pharmacy technician reported: "A woman used simvastatin. She stopped because of muscle pain. I asked her to contact her doctor. The patient agreed. In consultation with the doctor we reduced the dosage. The patient was very pleased that we thought about finding a solution."

5. Another pharmacy technician reported: "The use of the structured interview leads to insights into specific drug problems of the patient and helps to correctly apply the intervention."

Quotes regarding the communication with general practitioners

6. One pharmacist about informing general practitioners: "previous experience with doctors made me decide not to inform them. They do not want pharmacist's pharmaceutical care projects. They believe that we are trying to take their place. If necessary, I take their contact details because then I can call them about a specific problem of a certain patient."

Quotes related to promoting factors

7. One pharmacist reported: 'it is very important that pharmacists' assistants are involved in this intervention. It is very useful that they know what's going on in the patient, so that they can help."

8. Another pharmacist reported: "Patients tell a lot about themselves. Their information was valuable to me as a pharmacist. They indicated that they were pleased that we took our time. The patients had an opportunity to ask questions about their medicines and the problems they had taking them. This was good for the patients." Table 1: Quotes from pharmacists, pharmacy technicians and patients 
Adhien, P., Dijk, L. van, Vegter, M. de, Westein, M., Nijpels, G., Hugtenburg, J.G. Evaluation of an pilot study to influence medication adherence of patients with diabetes mellitus type-2 by the pharmacy. International Journal of Clinical Pharmacy: 2013, 35(6), 1113-1119

Table 2: Opinions of the patients about components of the intervention $(n=25)$

\begin{tabular}{|l|l|l|l|}
\hline Statements & $\begin{array}{l}\text { Strongly } \\
\text { disagree }\end{array}$ & $\begin{array}{l}\text { Neither disagree } \\
\text { nor agree }\end{array}$ & $\begin{array}{l}\text { Strongly } \\
\text { agree }\end{array}$ \\
\hline $\begin{array}{l}\text { I found it pleasant to be invited for } \\
\text { an interview via the phone }\end{array}$ & 1 & 4 & 20 \\
\hline $\begin{array}{l}\text { I appreciate being invited for an } \\
\text { interview in the pharmacy }\end{array}$ & & 4 & 21 \\
\hline \begin{tabular}{l|l|l|} 
I think that the proposed support is \\
important
\end{tabular} & 1 & 3 & 21 \\
\hline $\begin{array}{l}\text { I have the advantage of the advice } \\
\text { that I received }\end{array}$ & & 4 & 21 \\
\hline $\begin{array}{l}\text { I succeeded in following the advice } \\
\text { of the pharmacist }\end{array}$ & 1 & 2 & 22 \\
\hline
\end{tabular}

